\title{
A minimal physiologically based pharmacokinetic model for high-dose methotrexate
}

\author{
Giuseppe Pesenti ${ }^{1} \cdot$ Marco Foppoli $^{2} \cdot$ Davide Manca $^{1}$ (i)
}

Received: 31 December 2020 / Accepted: 30 May 2021 / Published online: 13 June 2021

(c) The Author(s) 2021

\begin{abstract}
Purpose High-dose methotrexate (HDMTX) is administered for the treatment of a variety of malignant tumors. Wide intraand inter-individual variabilities characterize the pharmacokinetics of MTX, which is mostly excreted renally. HDMTX dosages are prescribed as a function of body surface area whereas dose adjustments depending on renal function are not well defined. We develop a population pharmacokinetic model with a physiological description of renal excretion as the basis for clinical tools able to suggest model-informed dosages and support therapeutic monitoring.

Methods This article presents a minimal physiologically based pharmacokinetic (PBPK) model for HDMTX, which specifically accounts for individual characteristics such as body weight, height, gender, age, hematocrit, and serum creatinine to provide individualized predictions. The model supplies a detailed and mechanistic description of capillary and cellular exchanges between plasma, interstitial fluid, and intracellular fluid compartments, and focuses on an individualized description of renal excretion.

Results The minimal PBPK model is identified and validated with a literature dataset based on Chinese patients suffering from primary central nervous system lymphoma. A comparison with a pharmacokinetic model from the literature suggests that the proposed model provides improved predictions. Remarkably, the model does not present any significant bias in a wide range of degrees of renal function.

Conclusion Results show that model predictions can capture the wide intra- and inter-individual variability of HDMTX, and highlight the role played by the individual degree of renal function. The proposed model can be the basis for the development of clinical decision-support systems for individualized dosages and therapeutic monitoring.
\end{abstract}

Keywords Methotrexate $\cdot$ HDMTX $\cdot$ Pharmacokinetics $\cdot$ Minimal PBPK $\cdot$ Renal function

\section{Introduction}

Methotrexate (MTX) is an antifolate drug used to treat auto-immune disorders and in cancer chemotherapy. Highdose methotrexate (HDMTX) consists of doses higher than $500-1000 \mathrm{mg} / \mathrm{m}^{2}[1,2]$. HDMTX treats a variety of

Davide Manca

davide.manca@polimi.it

http://pselab.chem.polimi.it/

1 PSE-Lab, Process Systems Engineering Laboratory, Dipartimento di Chimica, Materiali e Ingegneria Chimica "Giulio Natta", Politecnico di Milano, Piazza Leonardo da Vinci 32, 20133 Milano, Italy

2 Unit of Lymphoid Malignancies, Division of Onco-Hematological Medicine, Department of Onco-Hematology, IRCCS San Raffaele Scientific Institute, Via Olgettina 60, 20132 Milano, Italy malignant tumors, which include primary central nervous system lymphoma (PCNSL), osteosarcoma, and acute lymphoblastic leukemia [3]. HDMTX is used to extend the exposure duration, overcome the mechanisms of resistance to MTX [4, 5], and penetrate the blood-brain barrier [6].

The administration of HDMTX is characterized by a very large intra- and inter-individual variability [7-9] and is associated with toxic side effects, with a reported incidence of HDMTX-induced nephrotoxicity that goes from 1.8\% [10] to $10.7 \%$ [11]. While there are several treatment protocols for each tumor type, they usually prescribe predetermined HDMTX dosages per square meter of body surface area (BSA) [2, 12-14].

MTX is mostly excreted through the kidneys [15] with creatinine clearance $(\mathrm{CrCl})$ being a strong determinant of methotrexate overall clearance $[5,16]$. Renal dysfunction leads to delayed MTX elimination and elevated plasma 
levels [10]. Despite this, adjustments for renal function are usually limited to the definition of cut-off values of either $\mathrm{CrCl}$ or glomerular filtration rate (GFR) for dose reduction or omission [1, 2, 4, 17]. Abrey [12] and Joerger and coauthors [16] report that uniform HDMTX dosing regimens are still lacking in the case of PCNSL, and suggest the introduction of personalized dosages to account for patient age, gender, and renal clearance, as they could significantly improve HDMTX treatment.

Few approaches to HDMTX dose adjustment have been published in the literature. Pignon et al. [18] proposed a dose adjustment approach to be performed during each 8-h cycle. Similarly, Evans et al. [19] showed that, in children with acute lymphoblastic leukemia, dose adjustments after $8 \mathrm{~h}$ of a $24 \mathrm{~h}$ MTX infusion lead to improved outcomes. Joerger et al. [9] proposed a dosing algorithm that uses the value of MTX concentration $24 \mathrm{~h}$ after the start of the first treatment cycle to adjust individual MTX doses in the following treatment cycles.

To the best of our knowledge, a decision-support tool capable of suggesting individualized HDMTX dosages for clinical use was not published in the literature. Accordingly, this article focuses on physiologically based pharmacokinetic (PBPK) modeling as the basis to develop that decisionsupport tool. Such a tool would also be suitable to support HDMTX therapeutic monitoring [20].

In 1970, Bischoff et al. [21] developed a remarkable pharmacokinetic model of methotrexate for mice, whose parameters attempted to maintain a good consistency with physiology. Then Zaharko et al. [22], Bischoff et al. [23], and Dedrick et al. [24] published developments of the same PBPK model for mice, other mammals, and humans. More recently, Ogungbenro et al. [25] proposed a methotrexate PBPK model for children and adults. However, these models do not account for individual characteristics such as body weight, height, gender, age, and degree of renal function, and their predictions of MTX pharmacokinetics can be directly applied only to a reference patient with standard body features.

We apply the general modeling approach proposed by Cao and Jusko [26] and present a new minimal PBPK model for the administration of intravenous HDMTX to adult patients that can account for individual characteristics such as body weight, height, gender, age, hematocrit, and renal function.

Model parameters are assigned as much as possible a priori and are functions of individual characteristics. The remaining parameters (which cannot be assigned a priori) are evaluated numerically via a non-linear regression against a literature dataset of patients suffering from PCNSL and via a bootstrap analysis. Our model adopts a populationPK approach, and the regressed parameters are assumed to constant for the whole population described.
The model devotes special attention to renal filtration. Consequently, it can be applied also to patients with reduced renal function. This approach allows improving the understanding of MTX pharmacokinetics, identifying the characteristics of peculiar populations, and refining the predictions of individual MTX pharmacokinetics.

\section{Methods}

\section{Mathematical model}

Figure 1 shows the overall structure of our minimal PBPK model, which was implemented in MATLAB R2020b (The MathWorks, Natick, MA, US). The model adopts the general minimal PBPK approach proposed by Cao and Jusko [26] and describes two lumped compartments for interstitial fluid (ISF) and intracellular fluid (ICF). The plasma circulation of the drug is described by dividing plasma into the renal circulatory system (RCS), the hepatic circulatory system (HCS), and the remaining part of the global circulatory system (CS). Each compartment is assumed homogeneous [27] so that in the generic compartment $j$ with volume $V_{j}$, the drug concentration $c_{j}$ can be calculated as $c_{j}=m_{j} / V_{j}$, where $m_{j}$ is the amount of drug.

The following system of ordinary differential equations (Eqs. 1-5) mathematically describes the drug material balances in the five model compartments (i.e. CS, RCS, HCS, ISF, and ICF) of Fig. 1:

$$
\begin{aligned}
\frac{d m_{C S}}{d t}= & \dot{m}_{I V}-Q_{C S \leftrightarrow R C S} c_{C S}+Q_{C S \leftrightarrow R C S} c_{R C S} \\
& -Q_{C S \leftrightarrow H C S} c_{C S}+Q_{C S \leftrightarrow H C S} c_{H C S} \\
& -\dot{m}_{C S \rightarrow I S F}+\dot{m}_{I S F \rightarrow C S}
\end{aligned}
$$

$\frac{d m_{R C S}}{d t}=Q_{C S \leftrightarrow R C S} c_{C S}-Q_{C S \leftrightarrow R C S} c_{R C S}-\dot{m}_{\text {excretion }}^{\text {renal }}$,

$\frac{d m_{H C S}}{d t}=Q_{C S \leftrightarrow H C S} c_{C S}-Q_{C S \leftrightarrow H C S} c_{H C S}-\dot{m}_{\text {elimination }}^{\text {hepatic }}$,

$\frac{d m_{I S F}}{d t}=\dot{m}_{C S \rightarrow I S F}-\dot{m}_{I S F \rightarrow C S}-\dot{m}_{I S F \rightarrow I C F}+\dot{m}_{I C F \rightarrow I S F}$,

$\frac{d m_{I C F}}{d t}=\dot{m}_{I S F \rightarrow I C F}-\dot{m}_{I C F \rightarrow I S F}$,

where $\dot{m}_{I V}$ is the MTX flow administered intravenously at each instant, $Q_{C S \leftrightarrow R C S}$ and $Q_{C S \leftrightarrow H C S}$ are the plasma flow rates exchanged with RCS and HCS, $\dot{m}_{C S \rightarrow I S F}$ and $\dot{m}_{I S F \rightarrow C S}$ represent the MTX flow rates exchanged between CS and ISF at the capillary level, $\dot{m}_{I S F \rightarrow I C F}$ and $\dot{m}_{I C F \rightarrow I S F}$ are the MTX 


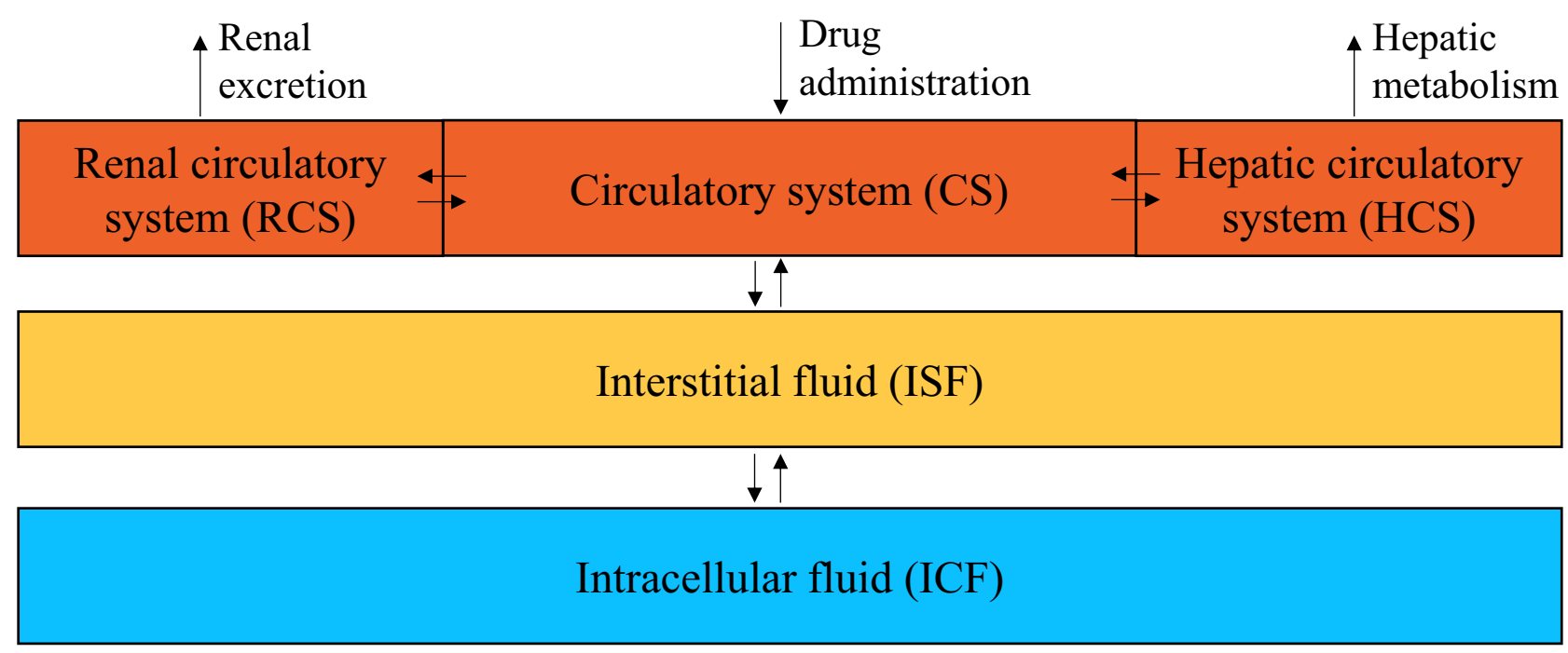

Fig. 1 Structure of the minimal PBPK model. The rectangles represent the compartments of the model: three plasma compartments (dark orange), the lumped ISF compartment (mustard), and the

flow rates entering and leaving ICF due to cellular exchange with ISF, $\dot{m}_{\text {excretion }}^{\text {renal }}$ is the renal excretion, and $\dot{m}_{\text {elimination }}^{\text {hepatic }}$ is the hepatic metabolic elimination. The model describes the capillary exchange between ISF and the plasma compartments as if it took place only within the global CS compartment.

\section{Plasma, ISF, and ICF volumes}

Assuming that the body volumes grow linearly with body weight, the reference values of $5.3 \mathrm{~L}$ blood volume for a $73 \mathrm{~kg}$ man and $3.9 \mathrm{~L}$ for a $60 \mathrm{~kg}$ woman [28] allow inferring that men and women have $72 \mathrm{~mL}$ and $65 \mathrm{~mL}$ of blood per $\mathrm{kg}$, respectively. Total plasma volume can be easily related to total blood volume by subtracting the hematocrit (HCT). The RCS and HCS plasma volumes are estimated, respectively, as $2 \%$ and $10 \%$ of total plasma [28]. Consequently, the CS plasma volume is the remaining fraction of plasma volume. The extracellular fluid (ECF) and ICF volumes are likewise estimated using as reference the average $\mathrm{ECW}$ and ICW values from Ritz et al. [29]. Finally, the ISF volume is calculated as the difference between ECF and plasma volumes.

Notably, our model does not fit the bodily volumes, and their values are instead assigned as individualized estimates from the patient's body weight and HCT. All the volume estimates refer to the physiological volumes of plasma, ISF, or ICF. It should be noted, however, that these values do not take into account the individual excess lumped ICF compartment (cyan). The arrows describe the drug flows, i.e. administration, exchange between compartments, metabolism, and excretion (colour figure online)

fat, nutrition, race, and age, and may further change according to, e.g. hydration and sodium balance.

\section{Plasma flow rates}

The total cardiac output (CO) $Q_{C O}^{\text {plasma }}$ (in $\mathrm{mL} / \mathrm{min}$ ) and the fractions of $\mathrm{CO}$ that reach the kidneys and the liver are estimated with the equations developed by Stader et al. [30] as a function of body weight, height, gender, age, and HCT.

\section{Methotrexate protein binding}

MTX is affected by protein binding, i.e. the reversible binding between the drug and blood proteins [31]. Protein binding of MTX occurs predominantly with serum albumin [32] and the drug is inactive while bound.

In plasma, reported values of MTX protein binding for healthy patients usually fall in the $42-57 \%$ interval [31] and appear to decrease in patients that are ill or in remission (e.g. to 34-40\% [33]). To apply the model to patients who suffer from tumor diseases and require the administration of HDMTX, the MTX bound fraction in plasma $f_{\text {plasma }}^{b}$ is assumed constant and equal to $42 \%$ [31]. Albumin gives rise to protein binding also in the ISF [34]. Following the approach of Schmitt [35], the MTX bound fraction in ISF $f_{I S F}^{b}$ is estimated as $28 \%$. Therefore, the complementary 
Table 1 List of model parameters

\begin{tabular}{|c|c|c|c|}
\hline Parameter & Description & Value & Units \\
\hline$V_{\text {plasma }}$ & Total plasma volume & Individualized & $\mathrm{mL}$ \\
\hline$V_{C S}$ & CS plasma volume & Individualized & $\mathrm{mL}$ \\
\hline$V_{R C S}$ & RCS plasma volume & Individualized & $\mathrm{mL}$ \\
\hline$V_{H C S}$ & HCS plasma volume & Individualized & $\mathrm{mL}$ \\
\hline$V_{I S F}$ & ISF volume & Individualized & $\mathrm{mL}$ \\
\hline$V_{I C F}$ & ICF plasma volume & Individualized & $\mathrm{mL}$ \\
\hline$Q_{C O}^{\text {plasma }}$ & Plasma cardiac output & Individualized & $\mathrm{mL} / \mathrm{min}$ \\
\hline$Q_{C S \leftrightarrow R C S}^{\text {plasma }}$ & Renal plasma flow (through renal arteries) & Individualized & $\mathrm{mL} / \mathrm{min}$ \\
\hline$Q_{C S \leftrightarrow H C S}^{\text {plasma }}$ & Hepatic plasma flow (through the hepatic vein) & Individualized & $\mathrm{mL} / \mathrm{min}$ \\
\hline$f_{\text {plasma }}^{b} / f_{\text {plasma }}^{u}$ & Bound/unbound MTX fraction in plasma & $0.42 / 0.58$ & - \\
\hline$f_{I S F}^{b} / f_{I S F}^{u}$ & Bound/unbound MTX fraction in ISF & $0.28 / 0.72$ & - \\
\hline$f_{I C F}^{b} / f_{I C F}^{u}$ & Bound/unbound MTX fraction in ICF & $0.9 / 0.1$ & - \\
\hline$Q_{G F R}$ & Glomerular filtration rate & Individualized & $\mathrm{mL} / \mathrm{min}$ \\
\hline$K_{50}^{\text {secretion }}$ & Secretion Michaelis constant & 1.3179E-04 & $\mathrm{mg} / \mathrm{mL}$ \\
\hline$\dot{m}_{\text {secretion }}^{\max }$ & Maximum rate of secretion & Individualized & $\mu \mathrm{g} / \mathrm{min}$ \\
\hline$K_{50}^{\text {reabsorption }}$ & Reabsorption Michaelis constant & $2.6358 \mathrm{E}-05$ & $\mathrm{mg} / \mathrm{mL}$ \\
\hline$\dot{m}_{\text {reabsorption }}^{\max }$ & Maximum rate of reabsorption & Individualized & $\mu \mathrm{g} / \mathrm{min}$ \\
\hline$Q_{\text {clerance }}^{\text {hepatic }}$ & Overall hepatic clearance & Individualized & $\mathrm{mL} / \mathrm{min}$ \\
\hline$K_{C S \rightarrow I S F}$ & $\mathrm{CS} \rightarrow \mathrm{ISF}$ exchange constant & Individualized & $\mathrm{mL} / \mathrm{min}$ \\
\hline$k_{C S \rightarrow I S F}$ & $\mathrm{CS} \rightarrow \mathrm{ISF}$ intensive exchange constant & Fitted & $\min ^{-1}$ \\
\hline$K_{I S F \rightarrow C S}$ & ISF $\rightarrow$ CS exchange constant & Individualized & $\mathrm{mL} / \mathrm{min}$ \\
\hline$k_{I S F \rightarrow C S}$ & ISF $\rightarrow$ CS intensive exchange constant & Fitted & $\min ^{-1}$ \\
\hline$K_{50}^{R F C, \text { influx }}$ & Cellular influx Michaelis constant & 3.6 & $\mu \mathrm{mol} / \mathrm{L}$ \\
\hline$\dot{m}_{I S F \rightarrow I C F}^{\max }$ & Maximum rate of cellular uptake & Individualized & $\mathrm{mg} / \mathrm{min}$ \\
\hline$k_{I S F \rightarrow I C F}^{\max }$ & Intensive maximum cellular uptake constant & Fitted & $\min ^{-1}$ \\
\hline$K_{50}^{R F C, \text { efflux }}$ & Cellular efflux Michaelis constant & 3.6 & $\mu \mathrm{mol} / \mathrm{L}$ \\
\hline$\dot{m}_{I C F \rightarrow I S F}^{\max }$ & Maximum rate of cellular efflux & Individualized & $\mathrm{mg} / \mathrm{min}$ \\
\hline$k_{I C F \rightarrow I S F}^{\max }$ & Intensive maximum cellular efflux constant & Fitted & $\min ^{-1}$ \\
\hline
\end{tabular}

MTX unbound fractions $f_{\text {plasma }}^{u}$ and $f_{I S F}^{u}$ are $58 \%$ and $72 \%$, respectively.

\section{Methotrexate renal excretion}

The main elimination route for MTX is renal excretion [2, 15]. Following intravenous administration of HDMTX, about $90 \%$ of the drug leaves the body unchanged through the urine within $154 \mathrm{~h}$ (i.e. almost a week) [36]. MTX is filtered by the renal corpuscles of each nephron, and undergoes active secretion and active reabsorption [15] as follows:

$\dot{m}_{\text {excretion }}^{\text {renal }}=\dot{m}_{\text {filtration }}+\dot{m}_{\text {secretion }}-\dot{m}_{\text {reabsorption }}$

Assuming that unbound MTX is freely filtered across the glomerular filtration barrier and neglecting the filtration of albumin-bound MTX, renal filtration is described as

$\dot{m}_{\text {filtration }}=Q_{G F R} c_{R C S} f_{\text {plasma }}^{u}$.
Several equations have been developed in the literature to estimate the individual $Q_{G F R}$ from the serum creatinine levels, since obtaining experimental creatinine values from the blood is cheap and convenient. These equations correlate the blood creatinine levels to the steady-state values of $Q_{G F R}$ for a specific population, also by taking into account the body weight, height, gender, and age of the patient. In this work, we employed the CKD-EPI equation with a 4-level variable for race [37], which is suitable to describe both patients with renal impairment and with normal renal function. We used the coefficients for Asians to describe Chinese patients. Additionally, the 97.5th percentile of the reference interval by the FAS equation [38] was used as the upper limit to the $Q_{G F R}$ estimates.

Secretion and reabsorption are described as a single overall saturable process (Eqs. 8 and 9) with Michaelis-Menten kinetics. The modeling of reabsorption is simplified and is expressed as a function of the drug concentration inside the plasma, similar to secretion. 


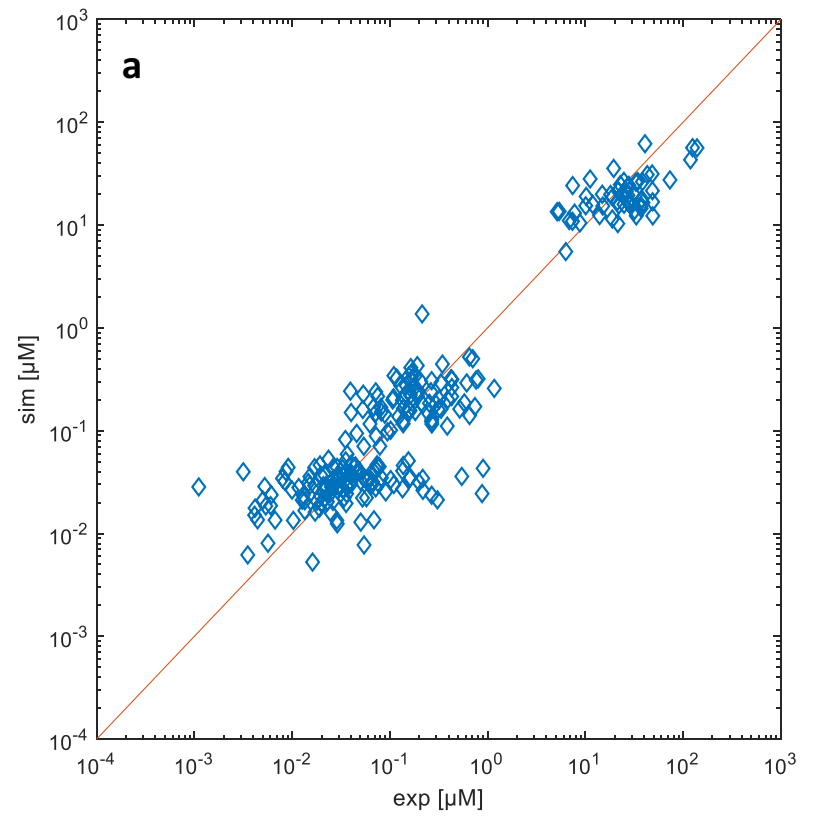

Fig. 2 Model predicted vs observed concentration plots for a our minimal PBPK model $(\mathrm{ObjFun}=0.8175)$ and $\mathbf{b}$ the 2-compartment pharmacokinetic model of Mei et al. [41] (ObjFun=1.0799). For

$\dot{m}_{\text {secretion }}=\dot{m}_{\text {secretion }}^{\max } \cdot \frac{c_{R C S}^{u}}{K_{50}^{\text {secretion }}+c_{R C S}^{u}}$,

$\dot{m}_{\text {reabsorption }}=\dot{m}_{\text {reabsorption }}^{\text {max }} \cdot \frac{c_{R C S}^{u}}{K_{50}^{\text {reabsorption }}+c_{R C S}^{u}}$.

The Michaelis constants $K_{50}^{\text {secretion }}$ and $K_{50}^{\text {reabsorption }}$ are estimated as $0.1 \mu \mathrm{mol} / \mathrm{L}$ and $0.6 \mu \mathrm{mol} / \mathrm{L}$, multiplied by $f_{\text {u,plasma }}$ [15], whereas $\dot{m}_{\text {secretion }}^{\text {max }}$ and $\dot{m}_{\text {reabsorption }}^{\text {max }}$ are assigned as 5.189 and $1.038 \mu \mathrm{g} / \mathrm{min}$ (for a reference $Q_{G F R}$ of $100 \mathrm{~mL} / \mathrm{min}$, assuming a proportionality to $Q_{G F R}$ ). The overall MTX renal clearance described exceeds that of creatinine by up to $15 \%$ in the $0.1-0.4 \mu \mathrm{mol} / \mathrm{L}$ range and is consistent with the experimental trends [15]. As MTX concentration increases, secretion and reabsorption reach saturation and their relative contribution becomes smaller, so that renal clearance approaches creatinine clearance and the glomerular filtration rate.

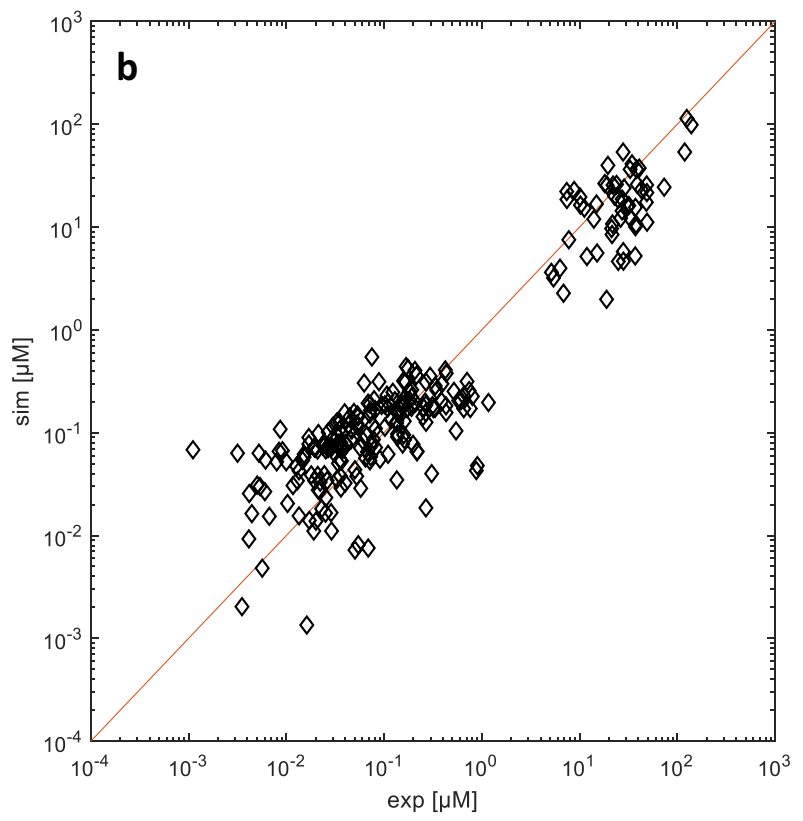

each experimental plasma concentration ( $x$-axis), the diagrams show the corresponding simulated value (y-axis)

\section{Methotrexate hepatic elimination}

MTX is affected by uptake into the hepatocytes of the liver, where it is metabolized into its major metabolite, 7-hydroxymethotrexate (7-OH-MTX), and is also affected by biliary secretion. In the intestinal tract, MTX can be reabsorbed into the systemic circulation, excreted through the feces, or hydrolyzed to 2,4-diamino-N10-methylpteroic acid (DAMPA), a minor metabolite [39]. These routes represent a minor contribution to the overall MTX elimination [40], which does not appear correlated to biomarkers of liver function [41]. In the case of HDMTX, since these routes depend mostly on saturable processes, it is likely that their relative importance decreases further. Hepatic processes are therefore described with a simplified approach, which neglects the enterohepatic circulation and assumes that MTX affected by hepatic clearance is immediately either metabolized into 7-OH-MTX or secreted into the bile and metabolized into DAMPA or eventually excreted through the feces (Eq. 10).
Table 2 Regressed parameters values

\begin{tabular}{llllll}
\hline Parameter & Investigated range & Initial value & Mean fitted value & Standard deviation & Units \\
\hline$k_{C S \rightarrow I S F}$ & 1.E-08-1.E0 & $2.1544 \mathrm{E}-02$ & $1.5782 \mathrm{E}-03$ & $2.3759 \mathrm{E}-04$ & $\mathrm{~min}^{-1}$ \\
$k_{I S F \rightarrow C S}$ & 1.E-08-1.E0 & $2.1544 \mathrm{E}-02$ & $5.1829 \mathrm{E}-03$ & $2.6271 \mathrm{E}-04$ & $\mathrm{~min}^{-1}$ \\
$k_{I S F \rightarrow I C F}^{\max }$ & $1 . \mathrm{E}-08-1 . \mathrm{E} 0$ & $3.1623 \mathrm{E}-07$ & $2.4088 \mathrm{E}-07$ & $2.9131 \mathrm{E}-08$ & $\mathrm{~min}^{-1}$ \\
$k_{I C F \rightarrow I S F}^{\max }$ & $1 . \mathrm{E}-08-1 . \mathrm{E} 0$ & $3.1622 \mathrm{E}-06$ & $5.6171 \mathrm{E}-06$ & $1.1798 \mathrm{E}-06$ & $\mathrm{~min}^{-1}$ \\
ObjFun & - & $1.2839 \mathrm{E} 0$ & $8.5684 \mathrm{E}-01$ & $1.0909 \mathrm{E}-01$ & - \\
\hline
\end{tabular}



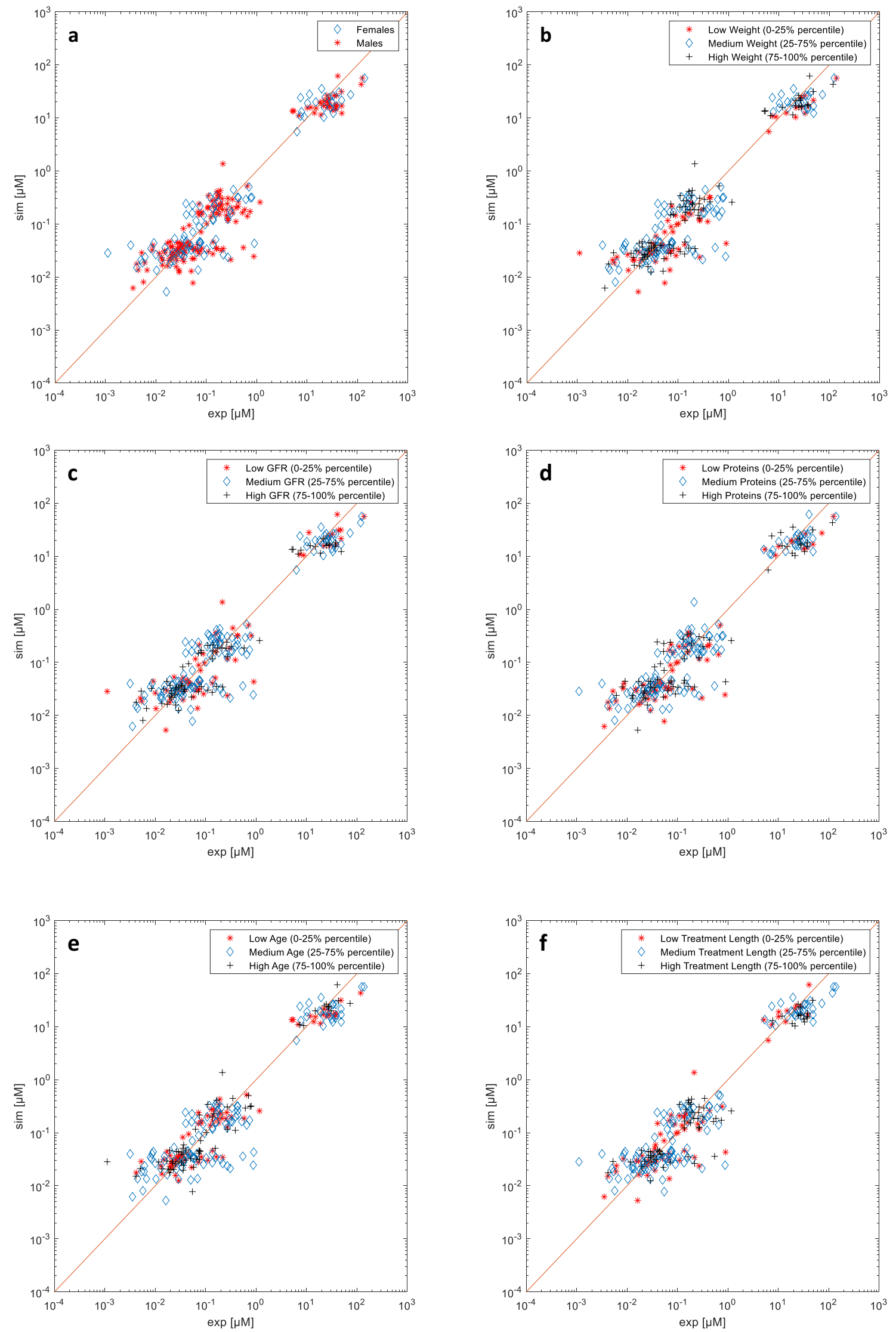
4Fig. 3 Model predicted vs observed concentration plots that highlight the conditions of each experimental point, according to $\mathbf{a}$ gender, $\mathbf{b}$ body weight, $\mathbf{c}$ GFR, $\mathbf{d}$ total blood proteins, e age, $\mathbf{f}$ length of treatment

$\dot{m}_{\text {elimination }}^{\text {hepatic }}=Q_{\text {clearance }}^{\text {hepatic }} c_{H C S}^{u}$.

The hepatic clearance is estimated as $4 \%$ of the GFR so that the overall hepatic elimination represents about $4 \%$ of the overall MTX clearance, approximately accounting for a $3 \%$ contribution from 7-OH-MTX metabolism [40] and 1\% from DAMPA metabolism and excretion through feces [15].

\section{Methotrexate capillary exchange}

MTX reaches the interstitial fluid through extravasation from the blood vessels at the capillary level. We describe capillary exchange in all tissues as generally permeabilitylimited (or diffusion-limited) [26, 42]. We assume that MTX is exchanged only by passive transport mechanisms, i.e. simple diffusion, according to the unbound $c_{C S}^{u}$ and $c_{I S F}^{u}$ concentrations:

$\dot{m}_{C S \rightarrow I S F}=K_{C S \rightarrow I S F} c_{C S}^{u}$,

$\dot{m}_{I S F \rightarrow C S}=K_{I S F \rightarrow C S} c_{I S F}^{u}$.

The exchange constants $K_{C S \rightarrow I S F}$ and $K_{I S F \rightarrow C S}$ represent the permeability-surface area product $P S(\mathrm{~mL} / \mathrm{min})$ and are estimated with Eq. (12) from their corresponding intensive constants $k_{C S \rightarrow I S F}$ and $k_{I S F \rightarrow C S}\left(\mathrm{~min}^{-1}\right)$.

$K_{C S \rightarrow I S F}=k_{C S \rightarrow I S F} V_{I S F}$,

$K_{I S F \rightarrow C S}=k_{I S F \rightarrow C S} V_{I S F}$.

Concerning the exchange between CS and ISF, at steadystate $\dot{m}_{C S \rightarrow I S F}=\dot{m}_{I S F \rightarrow C S}$ and, therefore,

$\frac{c_{I S F}^{u}}{c_{C S}^{u}}=\frac{K_{C S \rightarrow I S F}}{K_{I S F \rightarrow C S}}=\frac{k_{C S \rightarrow I S F}}{k_{I S F \rightarrow C S}}$,

$\frac{c_{I S F}}{c_{\text {plasma }}}=\frac{f_{\text {plasma }}^{u}}{f_{I S F}^{u}} \frac{k_{C S \rightarrow I S F}}{k_{I S F \rightarrow C S}}$.

By defining the tissue partition coefficient as $c_{I S F} / c_{\text {plasma }}$, its value is directly related with the $k_{C S \rightarrow I S F} / k_{I S F \rightarrow C S}$ ratio through $f_{\text {plasma }}^{u}$ and $f_{I S F}^{u}$ (Eq. 14). This equivalence allows expressing for both $k_{C S \rightarrow I S F}$ and $k_{I S F \rightarrow C S}$ the same physiological restrictions applied by Cao, Jusko [26] concerning CO. Furthermore, Eq. (13) shows that the $k_{C S \rightarrow I S F} / k_{I S F \rightarrow C S}$ ratio is related to the ratio of unbound concentrations at steadystate, which are expected to be equal [35, 43]. The values of $k_{C S \rightarrow I S F}$ and $k_{I S F \rightarrow C S}$, which are fitted, will account for the actual degree of permeability limitation in the capillary exchange and are expected to compensate for modeling errors, e.g. compartment volumes and protein binding.

\section{Methotrexate cellular exchange}

The minimal PBPK model is extended with an ICF compartment as MTX is affected by extensive cellular uptake [1]. Inside cells, methotrexate undergoes an extensive, reversible transformation from its native monoglutamate form into polyglutamate derivatives by folylpolyglutamate synthase (FPGS) [1, 44]. Our model does not provide an explicit description of the polyglutamate forms of methotrexate and neglects their efflux. Since Galivan [45] reports an intracellular polyglutamation exceeding $90 \%$, this value is tentatively taken as the reference value for the ICF bound fraction, described with $f_{I C F}^{b}$ and $f_{I C F}^{u}$ of about $90 \%$ and $10 \%$, respectively.

We assume that the total MTX uptake $\dot{m}_{I S F \rightarrow I C F}$ can be sufficiently described with a single Michaelis-Menten equation, representing the RFC (i.e. Reduced Folate Carrier [1]) transport system, as a function of the unbound ISF concentration:

$\dot{m}_{I S F \rightarrow I C F}=\dot{m}_{I S F \rightarrow I C F}^{\max } \cdot \frac{c_{I S F}^{u}}{K_{50}^{R F C \text { influx }}+c_{I S F}^{u}}$.

Similarly, Eq. (16) describes the total MTX efflux $\dot{m}_{I C F \rightarrow I S F}$ as a function of unbound ICF concentration:

$\dot{m}_{I C F \rightarrow I S F}=\dot{m}_{I C F \rightarrow I S F}^{\max } \cdot \frac{c_{I C F}^{u}}{K_{50}^{R F C, \text { efflux }}+c_{I C F}^{u}}$.

The Michaelis influx constant is approximately equal to $5 \mu \mathrm{M}$ [46], multiplied by $f_{I S F}^{u}$, and since RFC efflux shares significant similarities with RFC uptake [46], $K_{50}^{R F C \text {,efflux }}$ is assumed to be equal to $K_{50}^{R F C \text {,influx }}$.

Finally, $\dot{m}_{I S F \rightarrow I C F}^{\max }$ and $\dot{m}_{I C F \rightarrow I S F}^{\max }$ are the maximum rates exchanged between ISF and ICF and are assumed proportional to the total ICF volume (Eq. 17).

$\dot{m}_{I S F \rightarrow I C F}^{\max }=k_{I S F \rightarrow I C F}^{\max } V_{I C F}$,

$\dot{m}_{I C F \rightarrow I S F}^{\max }=k_{I C F \rightarrow I S F}^{\max } V_{I C F}$,

where the intensive parameters $k_{I S F \rightarrow I C F}^{\max }$ and $k_{I C F \rightarrow I S F}^{\max }\left(\min ^{-1}\right)$ are fitted.

Table 1 summarizes all the model parameters, detailing whether (i) they have a fixed assigned value, (ii) are estimated according to the individual features of the patient ("Individualized"), or (iii) are determined via a regression procedure ("Fitted"). 

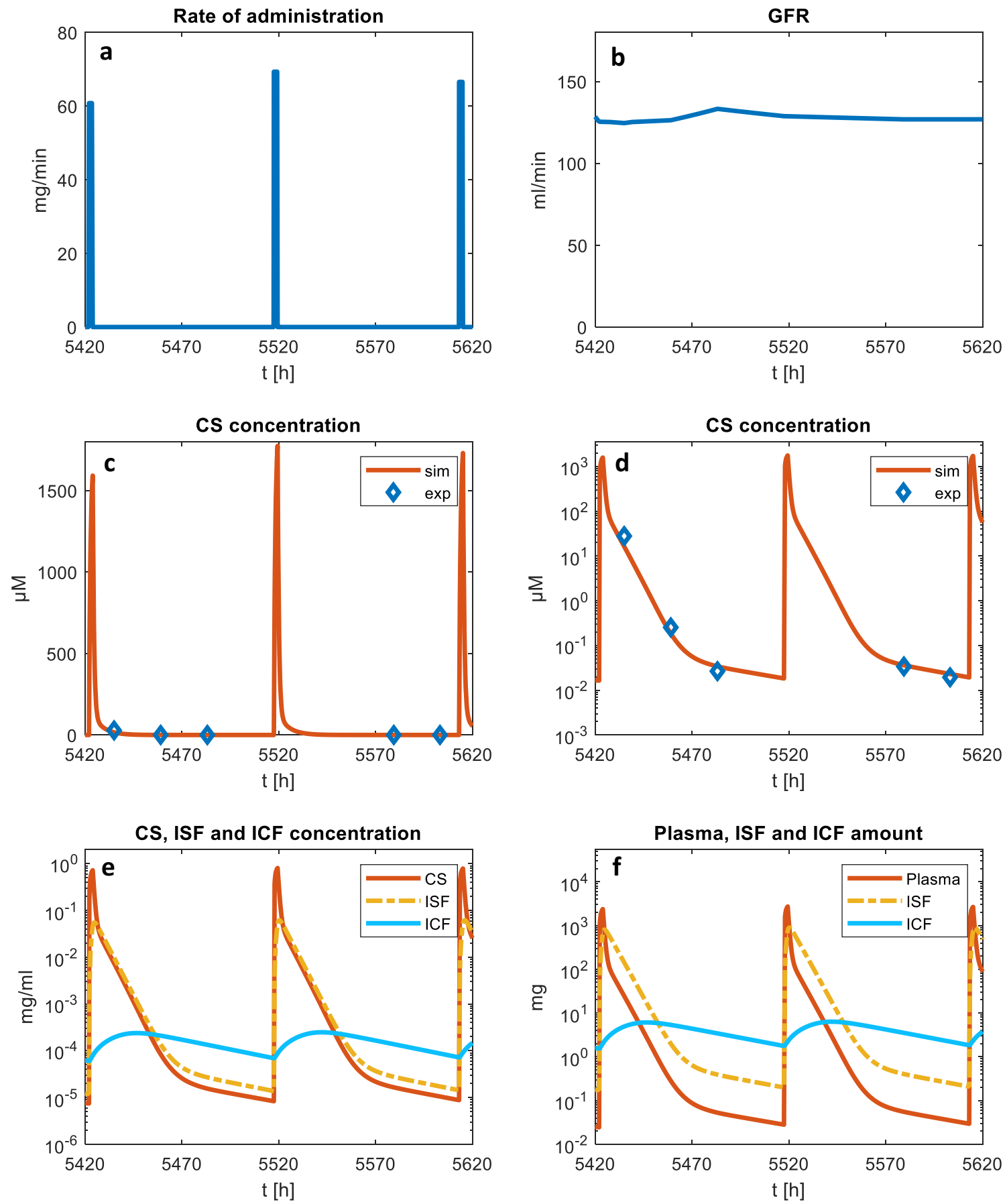

Fig. 4 Simulated curves for patient 630.3 with median error $(\mathrm{Obj}-$ Fun $=0.5416$ ): $\mathbf{a}$ infusion rates, b estimated GFR, c-d simulated and experimental CS concentrations (in linear and logarithmic coordi-

nates), e comparison of CS, ISF and ICF concentrations, $\mathbf{f}$ comparison of drug amounts in the overall plasma $(\mathrm{CS}+\mathrm{RCS}+\mathrm{HCS})$, ISF, and ICF

\section{Case study}

We employed the experimental dataset of Mei et al. [41] to perform the model identification and validation. The dataset collects the individual information of 98 Chinese patients with PCNSL who underwent intravenous HDMTX

treatment in Beijing Tiantan Hospital. A preliminary screening selected only the adults aged $\geq 18$ y of the study and allowed removing three outlier patients, i.e. patients labeled 580.1, 613.3, and 614.1, who showed plasma MTX concentrations higher than $0.2 \mu \mathrm{M}$ after more than $150 \mathrm{~h}$ following 
the administration of a 1.5-1.6 g dose. This led to the selection of 84 patients.

For each patient, Mei et al. [41] collected the body weight (44-100 kg), height (146-181 cm), gender (43 men, 41 women), age (27-83 y), data on comedications, as well as the results of laboratory tests such as hematocrit, serum creatinine, blood albumin, and total blood proteins. Most of the reported data were measured periodically. The population presents a wide range of degrees of renal function, as their estimated GFR values range from $35 \mathrm{~mL} / \mathrm{min}$ to $162 \mathrm{~mL} /$ $\min \left(39-150 \mathrm{~mL} / \mathrm{min} / 1.73 \mathrm{~m}^{2}\right)$, including patients with mild/ moderate renal impairment.

Patients received from 1 to 17 administrations of the drug, over a time period from $36 \mathrm{~h}$ to $352 \mathrm{~d}$, for a grand total of 396 infusions. The dataset reports the amount of each administration (1-9 g, or $\left.0.62-5.16 \mathrm{~g} / \mathrm{m}^{2}\right)$, the time of administration, and the infusion duration $(0.32-7.71 \mathrm{~h})$. We assume that the administered infusion rate was constant over the reported duration. Following administration, Mei et al. [41] measured plasma MTX concentrations every day, until the concentration fell below $0.05 \mu \mathrm{M}$, yielding 657 measurements $\left(N_{\text {tot }}^{\text {exp }}\right)$.

As discussed in the Methods section, the number of adaptive population parameters was kept as low as possible $\left(k_{C S \rightarrow I S F}, k_{I S F \rightarrow C S}, k_{I S F \rightarrow I C F}^{\max }, k_{I C F \rightarrow I S F}^{\max }\right)$. Their value is computed numerically (i.e. identified) through a non-linear regression procedure (Eq. 18, see [27]) against a subset of the experimental data [41] comprising 56 adult patients from Mei et al. [41]. These patients and the remaining twenty-eight patients feature similar distributions of body weight, gender, GFR, total blood proteins, and hematocrit.

$\min _{k_{C S \rightarrow I S F}, k_{I S F \rightarrow C S}, k_{I S F}^{\max } \rightarrow I C F}\left\{k_{I C F \rightarrow I S F}^{\max }\{\right.$ ObjFun $\}$.

We estimated ObjFun, the objective function, as the mean squared logarithmic errors between each experimental point $c_{C S, i j}^{\exp }$ and their corresponding simulated value $c_{C S, i j}^{s i m}$ :

ObjFun $=\frac{1}{N_{\text {tot }}^{e x p}} \sum_{i=1}^{N_{P}} \sum_{j=1}^{N_{i}^{e x p}}\left[\log \left(c_{C S, i j}^{e x p}\right)-\log \left(c_{C S, i j}^{\text {sim }}\right)\right]^{2}$.

\section{Results}

The regression procedure entailed a number of preliminary consecutive 4-dimension grid searches in a wide range of values (i.e. 1.E-08-1.E0), to identify a suitable starting point for an unconstrained minimization of the objective function. We carried out a 500 -sample bootstrap analysis by resampling the 56 patients of the identification dataset and running a non-linear regression for each bootstrap sample. We obtained an approximately normal distribution for each fitted parameter and identified their mean value and standard deviation (Table 2). The mean identified parameters lead to an objective function of 0.8568 for the identification dataset.

The identified parameters allow to numerically assess the $k_{C S \rightarrow I S F} / k_{I S F \rightarrow C S}$ ratio, which is equal to 0.3045 and might result from the compensation of errors of CS/ISF protein binding and volume estimates. The ratio agrees with the expected order of magnitude, i.e. around unity.

The simulation of the validation dataset featuring the remaining 28 patients yielded an objective function of 0.8175 (Fig. 2a), which is close to the one produced by the identification procedure $(0.8568)$.

These results are compared with the simulations of a 2-compartment pharmacokinetic model used in many literature MTX studies, applied by Mei et al. [41] to the same experimental dataset (Fig. 2b). For the same dataset used to validate our minimal PBPK model, the 2-compartment simulation yields an objective function of 1.0799 .

Figure 3a-f shows an analysis performed according to the specific conditions of the patients at each specific time, for sex, body weight, GFR, blood protein levels, age, and length of treatment. All the characteristic values are classified according to their distribution as low (0-25 percentiles), medium (26-75 percentiles), and high (76-100 percentiles).

The predicted overall MTX clearance, estimated as $\left(\dot{m}_{\text {excretion }}^{\text {renal }}+\dot{m}_{\text {elimination }}^{\text {hepatic }}\right) / c_{C S}^{u}$, approximately follows a normal distribution (mean $92.5 \mathrm{ml} / \mathrm{min}$, range 44-149 ml/min, coefficient of variation $21 \%$ ) for the 84 patients in the dataset.

Figure 4 shows the simulated curves for a specific section of the clinical data of patient 630.3 , whose objective function (0.5416) corresponds to the median value within the validation dataset. The 200 -h section includes three close, consecutive infusions and is deemed as representative of the whole dataset and useful to discuss the consistency of model predictions and their limitations.

Despite the wide range of experimental concentrations, as can be seen from both Fig. $4 \mathrm{c}$ and $\mathrm{d}$, the drug levels measured by Mei et al. [41] never capture the peak following HDMTX administration. In fact, while the drug administrations started between 9:45 AM and 11:15 PM, and finished in most cases before midnight (at most at 3:30 AM of the following day), the blood samples for MTX analysis were always taken between $5 \mathrm{AM}$ and $6 \mathrm{AM}$, i.e. hours later.

The model describes ICF concentrations that reach peaks of $0.2-0.3 \mu \mathrm{g} / \mathrm{mL}$, i.e. $0.44-0.66 \mu \mathrm{M}$. Indeed, the ICF compartment acts as an MTX reservoir that becomes significant when plasma MTX levels fall below $0.1 \mu \mathrm{M}$.

At concentrations lower than $0.1 \mu \mathrm{M}$, the simulations show a steady and very slow decrease of ICF concentration. As a result, the linear trend (in logarithmic coordinates of Fig. 4d) of plasma concentration is interrupted at about 
$0.1 \mu \mathrm{M}$ and a new quasi-linear (i.e. approximately exponential in linear coordinates) decrease can be observed, which is much slower, with a half time of about $40 \mathrm{~h}$.

\section{Discussion}

Four population parameters of the model are identified with a bootstrap analysis and non-linear regressions of individual experimental data that describe a population of patients suffering from PCNSL. The values of the $k_{C S \rightarrow I S F}$ and $k_{I S F \rightarrow C S}$ constants determine low $K_{C S \rightarrow I S F} / Q_{C O}^{\text {plasma }}$ fractions, in the $0.4-0.8 \%$ range, which suggests permeability-limited capillary exchange and distribution [26]. Despite its molecule being relatively small $(454 \mathrm{~g} / \mathrm{mol})$, MTX is hydrophilic and has limited lipid solubility [15]. As for $k_{I C F \rightarrow I S F}^{\max }$ and $k_{I S F \rightarrow I C F}^{\max }$, the maximum efflux rate $k_{I C F \rightarrow I S F}^{\max }$ is higher than the cellular uptake rate $k_{I S F \rightarrow I C F}^{\max }$, in agreement with [46].

The model is validated against a different set of patients from the same dataset. Figure 2 shows a wide distribution across the bisector. Indeed, the literature reports a very large intra- and inter-individual variability in the case of HDMTX treatment [7-9], and also Mei et al. [41] label this variability as "extremely large". Despite this, results suggest that the identified parameters are capable of capturing the average pharmacokinetic behavior of the diseased population. The comparison with a pharmacokinetic model used in several literature studies (Fig. 2b) also shows that, despite the rigid prior estimation of compartment volumes and clearance flows, the minimal PBPK model predictions appear to better account for the wide experimental variability of the dataset.

We investigated the model predictions against sex, weight, GFR, protein levels, or age (Fig. 3a-e) and found no indication of any clear systematic error. Remarkably, the model does not present any significant bias in a wide range of degrees of renal function, with estimated GFR values ranging from $35 \mathrm{~mL} / \mathrm{min}$ to $162 \mathrm{~mL} / \mathrm{min}(39 \mathrm{~mL} /$ $\mathrm{min} / 1.73 \mathrm{~m}^{2}$ to $150 \mathrm{~mL} / \mathrm{min} / 1.73 \mathrm{~m}^{2}$ ) (Fig. $3 \mathrm{c}$ ). This is expected to favor the application of the model to special populations with different characteristics, such as people with renal impairment. Conversely, when treatment length is medium (40-111 days) or high (111-325 days), model predictions appear to slightly underestimate the higher concentration values (Fig. 3f). This might indicate renal impairment arising with repeated administrations of HDMTX, as reported by [47]. Despite these findings, however, we did not impose a kidney function decline in our model. While the hypothesis of renal impairment is consistent with reports from the literature [47], the observed effect might be due to other factors, e.g. comedications such as dexamethasone, or also to a selection bias (patients who undergo longer treatments are ill patients who have not recovered yet despite multiple HDMTX cycles).

Finally, we described an intracellular fluid compartment characterized by slow saturable exchanges, which determines the prediction of a slow MTX decrease at low plasma concentrations (in the $0.001-0.1 \mu \mathrm{M}$ range). The overall predicted behavior appears generally consistent with the reference trend of MTX decay in Abelson et al. [48]. Further studies are recommended to improve the modeling of cellular uptake, efflux, and other intracellular phenomena.

We presented the development, identification, and validation of a minimal PBPK model of i.v. HDMTX methotrexate for adult patients. The model lumps the organs and tissues of the human body, and explicitly describes distinct compartments for plasma, interstitial fluid, and intracellular fluid. Since renal excretion is the main elimination route for methotrexate, the model is extended with a detailed description of renal excretion according to an anatomical and physiological approach. The model accounts for the specific characteristics and clinical data of each patient. Individual features such as body weight, height, gender, age, hematocrit, and serum creatinine are used to estimate a priori the pharmacokinetic parameters of each patient. This allows obtaining individualized predictions of MTX pharmacokinetics as a function of i.v. HDMTX administrations.

The model was developed using a dataset of Chinese patients with PCNSL and can be generalized to other populations by employing a suitable equation to estimate the GFR and by describing the different features of the target population, e.g. body volumes and protein binding.

Future developments should attempt to account for the residual intra- and inter-individual variability, especially by improving the estimation of volumes and exchanges as a function of further patient's features. In addition, model identification and validation should be replicated using different experimental datasets, possibly including patients with severe renal impairment, describing peak concentrations, and focusing on different populations and therapeutic protocols.

We believe that our model can be used to propose better individualized dosages and schedules of HDMTX, support the therapeutic monitoring of patients, and lead to better clinical treatment.

Acknowledgements The authors acknowledge the valuable contribution of Zhigang Zhao, Department of Pharmacy, Beijing Tiantan Hospital, Capital Medical University, Beijing, China, for his quick and helpful clarifications about the experimental dataset.

Funding Open access funding provided by Politecnico di Milano within the CRUI-CARE Agreement. 


\section{Declarations}

Conflict of interest The authors declare that they do not have any conflicts of interest.

Open Access This article is licensed under a Creative Commons Attribution 4.0 International License, which permits use, sharing, adaptation, distribution and reproduction in any medium or format, as long as you give appropriate credit to the original author(s) and the source, provide a link to the Creative Commons licence, and indicate if changes were made. The images or other third party material in this article are included in the article's Creative Commons licence, unless indicated otherwise in a credit line to the material. If material is not included in the article's Creative Commons licence and your intended use is not permitted by statutory regulation or exceeds the permitted use, you will need to obtain permission directly from the copyright holder. To view a copy of this licence, visit http://creativecommons.org/licenses/by/4.0/.

\section{References}

1. Joerger M, Huitema ADR, Illerhaus G, Ferreri AJM (2012) Rational administration schedule for high-dose methotrexate in patients with primary central nervous system lymphoma. Leuk Lymphoma 53(10):1867-1875. https://doi.org/10.3109/10428194. 2012.676177

2. Howard SC, McCormick J, Pui C-H, Buddington RK, Harvey RD (2016) Preventing and managing toxicities of high-dose methotrexate. Oncologist 21:1471-1482

3. Grommes C, DeAngelis LM (2017) Primary CNS lymphoma. J Clin Oncol 35(21):2410-2418. https://doi.org/10.1200/JCO.2017. 72.7602

4. Treon S, Chabner B (1996) Concepts in use of high-dose methotrexate therapy. Clin Chem 42(8):1322-1329

5. Comandone A, Passera R, Boglione A, Tagini V, Ferrari S, Cattel L (2005) High dose methotrexate in adult patients with osteosarcoma: clinical and pharmacokinetic results. Acta Oncol 44(4):406-411

6. Santisteban M, Nieto Y, De la Cruz S, Aristu J, Zubieta J, Hidalgo OF (2007) Primary central nervous system lymphoma treated with rituximab plus temozolomide in a second line schedule. Clin Transl Oncol 9(7):465-467

7. Fukuhara K, Ikawa K, Morikawa N, Kumagai K (2008) Population pharmacokinetics of high-dose methotrexate in Japanese adult patients with malignancies: a concurrent analysis of the serum and urine concentration data. J Clin Pharm Ther 33(6):677-684. https://doi.org/10.1111/j.1365-2710.2008.00966.x

8. Graf N, Winkler K, Betlemovic M, Fuchs N, Bode U (1994) Methotrexate pharmacokinetics and prognosis in osteosarcoma. J Clin Oncol 12(7):1443-1451. https://doi.org/10.1200/JCO.1994.12.7. 1443

9. Joerger M, Ferreri AJM, Krähenbühl S, Schellens JHM, Cerny T, Zucca E, Huitema ADR (2012) Dosing algorithm to target a predefined AUC in patients with primary central nervous system lymphoma receiving high dose methotrexate. Br J Clin Pharmacol 73(2):240-247. https://doi.org/10.1111/j.1365-2125.2011.04084.x

10. Widemann BC, Adamson PC (2006) Understanding and managing methotrexate nephrotoxicity. Oncologist 11(6):694-703. https:// doi.org/10.1634/theoncologist.11-6-694

11. Wiczer T, Dotson E, Tuten A, Phillips G, Maddocks K (2016) Evaluation of incidence and risk factors for high-dose methotrexate-induced nephrotoxicity. J Oncol Pharm Pract 22(3):430-436. https://doi.org/10.1177/1078155215594417
12. Abrey LE (2010) Hematology: individualized methotrexate dosing in primary CNS lymphoma. Nat Rev Clin Oncol 7(6):306

13. Ferreri AJM, Reni M, Foppoli M, Martelli M, Pangalis GA, Frezzato M, Cabras MG, Fabbri A, Corazzelli G, Ilariucci F, Rossi G, Soffietti R, Stelitano C, Vallisa D, Zaja F, Zoppegno L, Aondio GM, Avvisati G, Balzarotti M, Brandes AA, Fajardo J, Gomez H, Guarini A, Pinotti G, Rigacci L, Uhlmann C, Picozzi P, Vezzulli P, Ponzoni M, Zucca E, Caligaris-Cappio F, Cavalli F (2009) High-dose cytarabine plus high-dose methotrexate versus highdose methotrexate alone in patients with primary CNS lymphoma: a randomised phase 2 trial. The Lancet 374(9700):1512-1520. https://doi.org/10.1016/S0140-6736(09)61416-1

14. van den Bongard HD, Mathôt RA, Beijnen JH, Schellens JH (2000) Pharmacokinetically guided administration of chemotherapeutic agents. Clin Pharmacokinet 39(5):345-367

15. Grim J, Chládek J, Martínková J (2003) Pharmacokinetics and pharmacodynamics of methotrexate in non-neoplastic diseases. Clin Pharmacokinet 42(2):139-151. https://doi.org/10.2165/ 00003088-200342020-00003

16. Joerger M, Huitema A, Krähenbühl S, Schellens J, Cerny T, Reni M, Zucca E, Cavalli F, Ferreri A (2010) Methotrexate area under the curve is an important outcome predictor in patients with primary CNS lymphoma: a pharmacokinetic-pharmacodynamic analysis from the IELSG no. 20 trial. Br J Cancer 102(4):673

17. Kintzel PE, Dorr RT (1995) Anticancer drug renal toxicity and elimination: dosing guidelines for altered renal function. Cancer Treat Rev 21(1):33-64

18. Pignon T, Lacarelle B, Duffaud F, Guillet P, Catalin J, Durand A, Monjanel S, Favre R (1994) Pharmacokinetics of high-dose methotrexate in adult osteogenic sarcoma. Cancer Chemother Pharmacol 33(5):420-424. https://doi.org/10.1007/bf00686272

19. Evans WE, Relling MV, Rodman JH, Crom WR, Boyett JM, Pui C-H (1998) Conventional compared with individualized chemotherapy for childhood acute lymphoblastic leukemia. N Engl J Med 338(8):499-505. https://doi.org/10.1056/nejm19980219338 0803

20. Xu W-q, Zhang L-y, Chen X-y, Pan B-h, Mao J-q, Song H, Li J-y, Tang Y-m (2014) Serum creatinine and creatinine clearance for predicting plasma methotrexate concentrations after highdose methotrexate chemotherapy for the treatment for childhood lymphoblastic malignancies. Cancer Chemother Pharmacol 73(1):79-86

21. Bischoff KB, Dedrick RL, Zaharko DS (1970) Preliminary model for methotrexate pharmacokinetics. J Pharm Sci 59(2):149-154. https://doi.org/10.1002/jps.2600590203

22. Zaharko DS, Dedrick RL, Bischoff KB, Longstreth JA, Oliverio VT (1971) Methotrexate tissue distribution: prediction by a mathematical model. JNCI 46(4):775-784. https://doi.org/10. 1093/jnci/46.4.775

23. Bischoff KB, Dedrick RL, Zaharko DS, Longstreth JA (1971) Methotrexate pharmacokinetics. J Pharm Sci 60(8):1128-1133. https://doi.org/10.1002/jps.2600600803

24. Dedrick RL, Zaharko DS, Lutz RJ (1973) Transport and binding of methotrexate in vivo. J Pharm Sci 62(6):882-890. https://doi. org/10.1002/jps.2600620603

25. Ogungbenro K, Aarons L, CRESim T, Groups E-CP (2014) Physiologically based pharmacokinetic modelling of methotrexate and 6-mercaptopurine in adults and children Part 1 methotrexate. J Pharmacokinet Pharmacodyn 41(2):159-171. https://doi.org/10. 1007/s10928-014-9354-4

26. Cao Y, Jusko WJ (2012) Applications of minimal physiologicallybased pharmacokinetic models. J Pharmacokinet Pharmacodyn 39(6):711-723. https://doi.org/10.1007/s10928-012-9280-2

27. Abbiati RA, Lamberti G, Grassi M, Trotta F, Manca D (2016) Definition and validation of a patient-individualized 
physiologically-based pharmacokinetic model. Comput Chem Eng 84:394-408. https://doi.org/10.1016/j.compchemeng.2015. 09.018

28. Valentin J (2002) Basic anatomical and physiological data for use in radiological protection: reference values: ICRP Publication 89. Ann ICRP 32(3-4):1-277

29. Ritz P, Vol S, Berrut G, Tack I, Arnaud M, Tichet J (2008) Influence of gender and body composition on hydration and body water spaces. Clin Nutr 27(5):740-746

30. Stader F, Siccardi M, Battegay M, Kinvig H, Penny MA, Marzolini C (2019) Repository describing an aging population to inform physiologically based pharmacokinetic models considering anatomical, physiological, and biological age-dependent changes. Clin Pharmacokinet 58(4):483-501. https://doi.org/10.1007/ s40262-018-0709-7

31. Fiehn C (2010) Methotrexate transport mechanisms: the basis for targeted drug delivery and ss-folate-receptor-specific treatment. Clin Exp Rheumatol 28(5 Suppl 61):S40-45

32. Tirumalai RS, Chan KC, Prieto DA, Issaq HJ, Conrads TP, Veenstra TD (2003) Characterization of the low molecular weight human serum proteome. Mol Cell Proteomics 2(10):1096-1103. https://doi.org/10.1074/mcp.M300031-MCP200

33. Raveendran R, Heybroek W, Caulfield M, Lawson M, Abrams SM, Wrigley PF, Slevin M, Turner P (1992) Indomethacin and protein binding of methotrexate. Hum Exp Toxicol 11(4):291293. https://doi.org/10.1177/096032719201100411

34. Sloop CH, Dory L, Roheim PS (1987) Interstitial fluid lipoproteins. J Lipid Res 28(3):225-237

35. Schmitt W (2008) General approach for the calculation of tissue to plasma partition coefficients. Toxicol In Vitro 22(2):457-467. https://doi.org/10.1016/j.tiv.2007.09.010

36. Christophidis N, Louis WJ, Lucas I, Moon W, Vajda FJ (1981) Renal clearance of methotrexate in man during high-dose oral and intravenous infusion therapy. Cancer Chemother Pharmacol 6(1):59-64

37. Stevens LA, Claybon MA, Schmid CH, Chen J, Horio M, Imai E, Nelson RG, Van Deventer M, Wang H-Y, Zuo L, Zhang YL, Levey AS (2011) Evaluation of the chronic kidney disease epidemiology collaboration equation for estimating the glomerular filtration rate in multiple ethnicities. Kidney Int 79(5):555-562. https://doi.org/ 10.1038/ki.2010.462

38. Pottel H, Delanaye P, Weekers L, Selistre L, Goffin K, Gheysens O, Dubourg L (2017) Age-dependent reference intervals for estimated and measured glomerular filtration rate. Clin Kidney J 10(4):545-551. https://doi.org/10.1093/ckj/sfx026
39. Widemann BC, Sung E, Anderson L, Salzer WL, Balis FM, Monitjo KS, McCully C, Hawkins M, Adamson PC (2000) Pharmacokinetics and metabolism of the methotrexate metabolite 2,4-diamino-N10-methylpteroic Acid. J Pharmacol Exp Ther 294(3):894-901

40. Seideman P, Beck O, Eksborg S, Wennberg M (1993) The pharmacokinetics of methotrexate and its 7-hydroxy metabolite in patients with rheumatoid arthritis. Br J Clin Pharmacol 35(4):409-412. https://doi.org/10.1111/j.1365-2125.1993.tb041 58.x

41. Mei S, Li X, Jiang X, Yu K, Lin S, Zhao Z (2018) Population pharmacokinetics of high-dose methotrexate in patients with primary central nervous system lymphoma. J Pharm Sci 107(5):1454-1460. https://doi.org/10.1016/j.xphs.2018.01.004

42. Evans MV, Dowd SM, Kenyon EM, Hughes MF, El-Masri HA (2008) A physiologically based pharmacokinetic model for intravenous and ingested dimethylarsinic acid in mice. Toxicol Sci 104(2):250-260. https://doi.org/10.1093/toxsci/kfn080

43. Poulin P, Theil FP (2000) A priori prediction of tissue: plasma partition coefficients of drugs to facilitate the use of physiologicallybased pharmacokinetic models in drug discovery. J Pharm Sci 89(1):16-35. https://doi.org/10.1002/(SICI)1520-6017(200001) 89:1\%3c16::AID-JPS3\%3e3.0.CO;2-E

44. Inoue K, Yuasa H (2014) Molecular basis for pharmacokinetics and pharmacodynamics of methotrexate in rheumatoid arthritis therapy. Drug Metab Pharmacokinet 29(1):12-19. https://doi.org/ 10.2133/dmpk.DMPK-13-RV-119

45. Galivan J (1979) Transport and metabolism of methotrexate in normal and resistant cultured rat hepatoma cells. Can Res 39(3):735

46. Matherly LH, Goldman D (2003) Membrane transport of folates. Vitam Horm 66:405-457

47. Green MR, Chamberlain MC (2008) Renal dysfunction during and after high-dose methotrexate. Cancer Chemother Pharmacol 63(4):599. https://doi.org/10.1007/s00280-008-0772-0

48. Abelson H, Fosburg M, Beardsley G, Goorin A, Gorka C, Link M, Link D (1983) Methotrexate-induced renal impairment: clinical studies and rescue from systemic toxicity with high-dose leucovorin and thymidine. J Clin Oncol 1(3):208-216

Publisher's Note Springer Nature remains neutral with regard to jurisdictional claims in published maps and institutional affiliations. 